\title{
Low temperature Atomic Force Microscopy-A Review
}

P.M. Bhatt ${ }^{1}$, U.S.Joshi ${ }^{2}$ H.N.Shah ${ }^{3}$, P.K.Brahmbhatt ${ }^{4}$

${ }^{1}$ Mechanical Engineering Department, R.K.University, Rajkot, India

${ }^{2}$ Department of Physics, University School of Sciences, Gujarat University, Ahmedabad, India

${ }^{3}$ Department of Mechanical Engineering, Sakalchand Patel College of Engineering, Visanagar, India

${ }^{4}$ Department of Mechanical Engineering, Government Engineering College, Modasa,India

\begin{abstract}
Atomic force microscope (AFM) is successfully utilized to image a broad range of thin film specimens because it can image nonconductive and soft material. Since the invention of the AFM, a lot of progress has been made in imaging thin films. Operation at low temperatures brings the benefits of low thermal drift and low thermal noise, which are required for high-resolution measurements. Due to all these benefits cryogenic AFM was developed. The system operates at liquid nitrogen temperatures, and aims to reduce the main intrinsic resolution reducing effects of AFM imaging by freezing samples and imaging them at sub zero temperatures. Further studies on biomolecules and the effects of sub zero temperatures, especially of ice formation on biomolecules structure need to be carried out before this system is routinely used for molecular imaging. The surface morphology of the thin films of $\mathrm{In}_{2} \mathrm{O}_{3}, \mathrm{ITO}$ and PCMO were studied by Atomic Force Microscopy. AFM showed good surface morphology with decreasing RMS area roughness and Line roughness for all above three materials.
\end{abstract}

Keywords: Atomic Force Microscope, Vacuum Chamber, $\mathrm{LN}_{2}$ Reservoir, Thin Film

\section{Introduction}

The atomic force microscope (AFM) was invented by Binning et al. in 1986. The AFM measures the forces acting between a fine tip and a sample. The tip is attached to the free end of a cantilever and is brought very close to a surface. Attractive or repulsive forces resulting from interactions between the tip and the surface will cause a positive or negative bending of the cantilever ${ }^{[1,2,3]}$. The bending is detected by means of a laser beam, which is reflected from the backside of the cantilever.

The remarkable feature of Scanning Probe Microscopes (SPM) is their ability to view details at the atomic and molecular level, thus increasing our understanding of how systems work and leading to new possibility of exploring the different outcomes in many fields. These include life science, materials science, electrochemistry, polymer science, biophysics, nanotechnology, biotechnology, and many more ${ }^{[1,2]}$.

The principal behind the operation of an AFM in the contact mode is shown in Figure 1. The AFM tip is first brought close to the sample surface, and then the scanner makes a final adjustment of distance between cantilever tip and sample surface. The tip, now in contact with the sample surface is scanned across the sample under the action of a piezoelectric actuator. A laser beam aimed at the back of the cantilever-tip assembly reflects off the cantilever surface to a split photodiode, which detects the small cantilever deflections. A feedback loop, maintains constant tip-sample separation by moving the scanner in the $\mathrm{z}$ direction. Without this feedback loop, the tip would "crash" into a sample with even small topographic features ${ }^{[4,5]}$.

Atomic force microscopy is currently applied to various environments (air, liquid, vacuum) and types of materials such as metal semiconductors, soft biological samples, conductive and non-conductive materials. With this technique size measurements or even manipulations of nano-objects may be performed.AFM has the advantage that the sample preparation procedures are relatively simple and convenient, and also staining and/or coating is not necessary. While many research groups have reported imaging of biological materials in air or in fluid ${ }^{[6]}$ there have been few reports of freeze- fracture surface observations. This is very likely because there are few AFM that are suitable for biological samples, i.e., that can operate below freezing without significant ice formation ${ }^{[7]}$. The aim of this review articles are to present possible experimental setup of an AFM chamber for the investigation of biological freeze-fracture surfaces. 


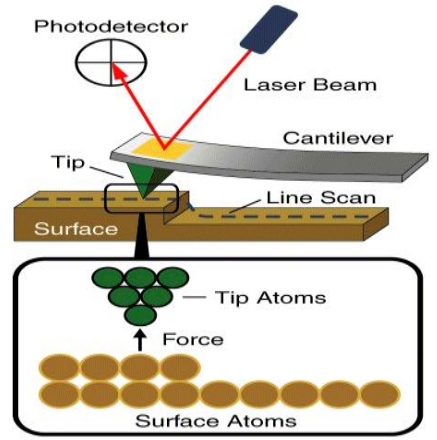

Fig:1 Principal Of Atomic Force Microscope

\section{History and background}

AFM provides a number of advantages over conventional microscopy techniques. AFM explores the sample and make measurements in three dimensions, $\mathrm{x}, \mathrm{y}$, and $\mathrm{z}$ (normal to the sample surface), thus enabling the presentation of three-dimensional images of a sample surface. This provides a great advantage over any microscope available previously. With good samples (clean, with no excessively large surface features), resolution in the $\mathrm{x}-\mathrm{y}$ plane ranges from 0.1 to $1.0 \mathrm{~nm}$ and in the $\mathrm{z}$ direction is $0.01 \mathrm{~nm}$ (atomic resolution). With these advantages AFM has significantly impacted the fields of materials science, chemistry, biology, physics, and the specialized field of semiconductors ${ }^{[4]}$.

Despite the success of the STM, it has a limitation. Because it uses the tunneling current between a biased tip and a sample as the feedback signal, it requires that the sample material must be electrically conductive. And even conductors (conductive metals and semiconductors) cannot be studied in ambient conditions by STM except for a few special materials, like highly oriented pyrolytic graphite (HOPG). Instead they have to be investigated in an ultrahigh vacuum because the surface layer of solids constantly changes by adsorption and desorption of atoms and molecules in ambient conditions ${ }^{[8]}$.

Operation at low temperatures brings the benefits of low thermal drift and low thermal noise, which are required for high-resolution measurements. On the other hand, very low temperature refrigeration techniques often hamper the efforts to achieve high-resolution measurements due to the introduction of mechanical vibrations. Furthermore, the physical space within the cryostat, especially when a high magnetic field is required, is often too limited to allow an effective cryogenic vibration-isolation stage. Our efforts to address these challenges are concentrated mainly in three elements: a very rigid STM head that is less susceptible to vibration, a refrigeration scheme that has very low intrinsic vibration noise, and a good external vibrationisolation system to reduce transmission of vibrations from the external environment to the STM cryostat ${ }^{[9]}$.

\section{Cryostat Method ${ }^{[10}$}

\section{Different Methods to Develop Low Temperature:}

Atomic force microscopy has become an important tool for studying surfaces at the atomic scale in real space due to the possibility of imaging insulating surfaces. To enhance the stability of the AFM many researchers have developed low temperature instruments. The development of such instruments is challenging, but crucial advantages such as diminished piezohysteresis and creep, reduced thermal drift, and lower noise level make it desirable.

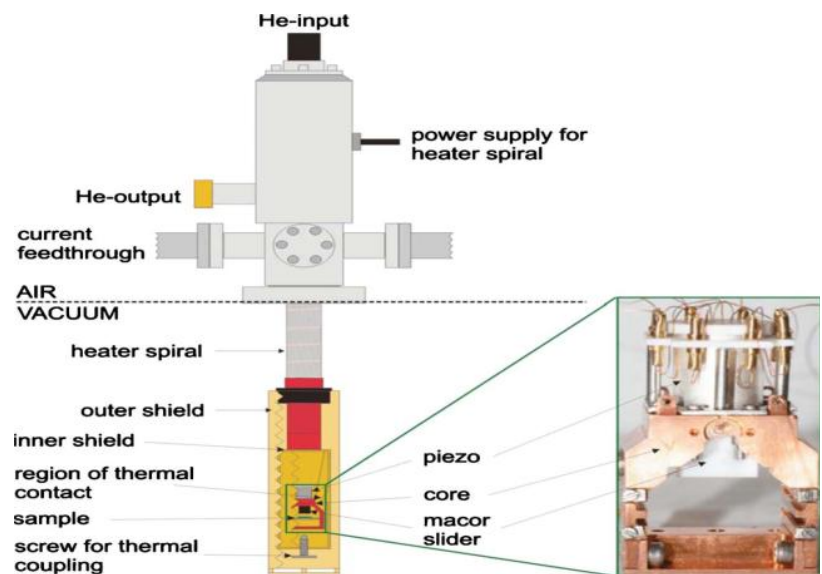

Fig: 3.1 Low Temperature-AFM and the flow cryostat 
In this method the microscope is built in the form of a copper cylinder with a diameter of only $3 \mathrm{~cm}$ and a height of $10 \mathrm{~cm}$. It exhibits two shields to reflect heat radiation and ensure that the sample and the tip stay clean by cryopumping. The shields are connected to a commercial flow cryostat (type APD Cryogenics LT-3B). The cryostat cools the microscope by thermal contact and can be operated either with liquid nitrogen or with liquid helium. Temperatures as low as $7 \mathrm{~K}$ can be reached as measured in close proximity to the sample. By simultaneous heating the cryostat the AFM can be stabilized at temperatures between $7 \mathrm{~K}$ and room temperature.

\section{Helium Cryostate ${ }^{[11]}$}

To design an STM this can achieve atomic resolution while operating at very low temperatures is a challenging task. Operation at low temperatures brings the benefits of low thermal drift and low thermal noise, which are required for high-resolution measurements. On the other hand, very low temperature refrigeration techniques often hamper the efforts to achieve high-resolution measurements due to the introduction of mechanical vibrations. Furthermore, the physical space within the cryostat, especially when a high magnetic field is required, is often too limited to allow an effective cryogenic vibration-isolation stage. Our efforts to address these challenges are concentrated in the following three elements:

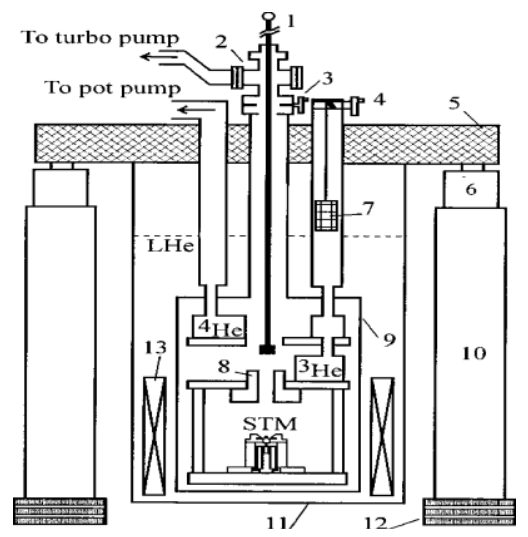

Fig: 3.2 STM Cryostat And Vibration-Isolation.

The refrigeration scheme used in this setup is a specially designed, low noise, single shot, 3He sorption refrigerator. As shown in the schematic drawing (Fig. 3.2), the refrigerator is housed in a vacuum can which is immersed in a liquid helium bath inside a super insulated Dewar. The small (4.5 in. diameter) but long (3 ft.) neck of the Dewar reduces the boil-off rate of the liquid helium to about 4litre a day with the super-conducting magnet installed! And thus also reduces vibrations due to evaporation of the liquid 4He in the bath. A 750 litre per min rotary pump is used to pump on the $4 \mathrm{He}$ pot, which is inside the vacuum can, to achieve a temperature of $1.2 \mathrm{~K}$ at the $4 \mathrm{He}$ stage. This temperature will condense the $3 \mathrm{He}$ sample into about $10 \mathrm{cc}$ of liquid in the $3 \mathrm{He}$ pot. When lowered into the $4.2 \mathrm{~K}$ region by a lift mechanism pumps on the condensed $3 \mathrm{He}$ to achieve a base temperature of $220 \mathrm{mK}$ at the $3 \mathrm{He}$ stage.

\section{$\mathbf{L N}_{2}$ Method (Cold Finger Concept) ${ }^{[9]}$}

In this experiment modified a commercial AFM For this experiment. The AFM head contains the following components, all of which are located inside the vacuum chamber: laser diode, four-quadrant photodiode, and preamplifier. The cantilever's deflection is detected using the optical beam bounce technique. The laser is aligned before evacuation. The head and thus the position of the cantilever are held constant during observation. The scanner is a tube type piezo with a maximum scan range of $80-\mu \mathrm{m} \times 380 \mu \mathrm{m} \times 35 \mu \mathrm{m}$. The scanner is mounted to the $\mathrm{XYZ}$ coarse alignment stage. This stage is for positioning the sample under the tip and can be adjusted from outside the vacuum chamber to locate a feature of interest other than the sample that has been freeze-fractured.

The copper cold finger coupled to an LN2 Dewar is mounted to the AFM chamber as shown in Fig.3.3. The sample stage can then be cooled via a heat conductor fabricated from ultra pure copper foils, where one end of the heat conductor is connected to the sample stage while the other end is coupled to the cold finger. The copper foils serve not only to transmit heat but also to decouple the vibration due to LN2 boiling. The copper foils also allow for the coarse stage motion as well as for scanning with the piezo scanner. The sample stage can be heated from its terminal temperature of $-175-0{ }^{\circ} \mathrm{C}$ by a heating resistor $(47 \mathrm{~V}, 0.125 \mathrm{~W})$. It is possible to heat the sample to room temperature with the heating resistor. The temperature of the sample stage can be monitored by a platinum thin film thermistor. 
The temperature of the sample holder can be controlled in the range from -175 to $0{ }^{\circ} \mathrm{C}$ using a commercially available proportional integral differential temperature controller that regulates the power dissipated in the resistor. The accuracy of the temperature adjustment is $0.1{ }^{\circ} \mathrm{C}$. The sample holder is thermally isolated from the piezo tube by an epoxy pillar heat shield.

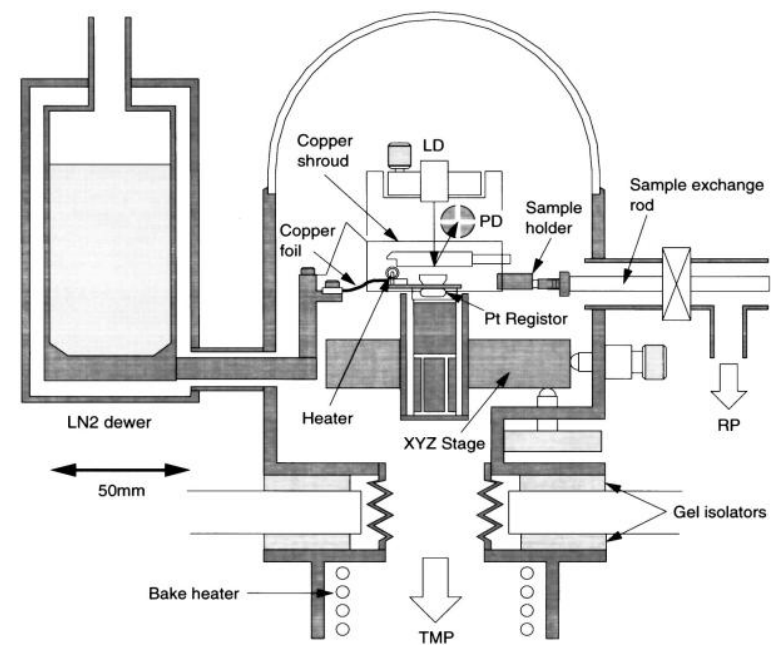

Fig. 3.3 Cross sectional drawing of the AFM.

A gold-coated copper shroud is mounted around the sample holder. The end of the copper shroud is coupled to the LN2 cooled cold finger. The shroud temperature is kept at $-150^{\circ} \mathrm{C}$ during cooling.

\section{Low Temperature Atomic Force Microscope ${ }^{[12,13]}$}

Figure 3.4 shows that sample holder is cooled via copper foils that are connected to a liquid nitrogen Dewar. The AFM use the optical beam bounce detection scheme and the piezo tube scanner is located on the XYZ coarse stage. A sample exchange rod transfers the sample holder stub. The instrument is evacuated by a 3001/s turbo molecular pump.

Fabrication of equipment for providing low temperature to for application of SPM thin film surfaces is a huge technological importance in modern science technology due to its variety of application in Physics, Polymer, Biological and medical science. The present work is a comprehensive study of thin film at low temperature. The characterizations of films were done by Atomic Force Microscopy method.

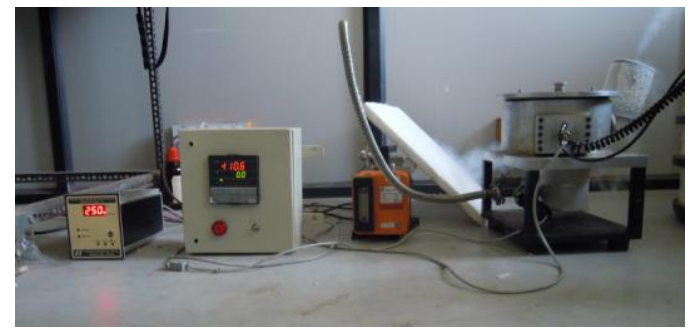

Fig: 3.4 Set up of Low temperature AFM

Liquid Nitrogen Vessel has been designed with safe pressure and during experiment it has been observed that no trouble is due to $\mathrm{LN}_{2}$ vessel. Copper rod is used as a cold finger concept. Copper has characteristic that with decreasing temperature thermal conductivity of copper is increased, which help to getting low temperature. Hollow copper rod is used instead of solid rod. Solid rod was taken more time to cool down due to higher inertia. Almost $137 \mathrm{~K}$ temperatures achieved within 7 minutes, while using hollow copper rod. At the same time only 223K temperatures has been achieved by Solid copper rod. Percentage error of Boil off rate is around $11 \%$, which can be reduced by using multilayer insulation around inner vessel. Even with using Teflon cover at the top of the flange boil off rate has been reduced. The surface morphology of the thin films of $\mathrm{In}_{2} \mathrm{O}_{3}$, ITO and PCMO were studied by Atomic Force Microscopy. AFM showed good surface morphology with decreasing RMS area roughness and Line roughness for all above three materials. The films exhibit a finer microstructure and consisted of smaller and more uniform grains forming a rather flat but more complex surface texture which is consistent with less rough topography. The results indicate that there is a scope for achieving better morphology and structure formation using still low temperature. 


\section{Conclusion}

The sample stage can be cooled $\mathrm{Cu}$ foil heat conductor, which is connected to a liquid nitrogen Dewar. Almost $137 \mathrm{~K}$ temperatures achieved within 7 minutes, while using hollow copper rod. At the same time only $223 \mathrm{~K}$ temperatures has been achieved by Solid copper rod. A very compact design AFM which can operate at variable temperature down to $7 \mathrm{~K}$ while using Helium. The films exhibit a finer microstructure and consisted of smaller and more uniform grains forming a rather flat but more complex surface texture which is consistent with less rough topography as well as spectroscopic data were measured at room temperature as well as at low temperature.

\section{Acknowledgement:}

The author's acknowledged fund support from Department of Science and Technology, Government of India under the DST-FIST(Level-1) support to produce the AFM set-up.

\section{Reference:}

[1] Arantxa Vilalta-Clemente "Principles of Atomic Principles Force Microscopy" ,Physics of Advanced Materials Winter School 2008, PP.1-10

[2] Binnig, G.; Quate, C. F.; Gerber, Ch., Phys. Rev. Lett. ,1986,PP.56, 930.

[3] Xiaogang Liu, and Hua Zhang., "Scanning Probe Microscopy-Based Nanofabrication for Emerging Applications", 2007

[4] C. Debuschewitz, F. M"unstermann, V. Kunej, and E. Scheer "A Compact and Versatile Scanning Tunnelling Microscope with High Energy Resolution for Use in a 3He Cryostat” Journal of Low Temperature Physics, Vol. 147, Nos. 3/4, May 2007 ,PP.525535

[5] D. Erts, A. Lõhmus, R. Lõhmus, H. Olin "Instrumentation of STM and AFM combined with transmission electron microscope" Third International Conference on Non-Contact Atomic Force Microscopy, July 16-19, 2000, Hamburg, Germany PP.S71-S74

[6] Alexandra Radenovic', "A low-temperature ultrahigh vacuum atomic force microscope for biological applications", Review Of Scientific Instruments Volume 74 ,2002,,PP.1022-1025

[7] Ian James Turner, "AFM investigations of critical interactions in the Bacillus primosome and Cryogenic AFM a new tool for structural biology", Thesis submitted to The University of Nottingham for the degree of Doctor of Philosophy,2005, PP.136-153

[8] Changyi Li “Cryogenic Atomic Force Microscope For Characterization Of Nanostructures"Thesis submitted to the Faculty of the Graduate School of the University of Maryland,2005, PP.1-49

[9] Keiichi Nakamoto, C. B. Mooney and Masashi Iwatsuki "Development of low-temperature and high vacuum atomic force microscope with freeze-fracture function"Review Of Scientific Instruments Volume 72-2, 2001 ,PP.1445-1448

[10] N. Wintjes, M. Lange, D. van Vörden, H. Karacuban, D. Utzat, and R. Möllera "Very compact design for a low-temperature tuning fork atomic force Microscope American Vacuum Society", 2010, PP.C4E21- C4E24

[11] S. H. Pan, E. W. Hudson, and J. C. Davis, "3He refrigerator based very low temperature scanning tunneling Microscope", Review Of Scientific Instruments Volume 70-21999, PP.1459-1463

[12] P.M. Bhatt, D.H. Agrawal, A.M. Pathan, N.D.Chauhan "In2o3 thinfilm Analysis by Low Temperature Atomic Force Microscope"Science Direct Procedia Enginnering 00(2011)000-000

[13] D.H.Agrawal,P.M.Bhatt,A.M.Pathan,Hitarthi Patel,U.S.Joshi "Development of Portable Experimental set-up for AFM to work at Cryogenic Temperature"AIP.Conf.Proc.1447,531(2012);doi:10.1063/1.4710113

[14] CHERYL R. BLANCHARD Atomic Force Microscopy VOL . 1, N O . 5 I SSN 1430 - 4171 THE C H EMICAL EDUCATOR @ 1996 SPRI NGER- VERLAG NEW YORK, I NC.

[15] M Heyde, G Thielsch, H-P Rust and H-J Freund 2005 A reverse pendulum bath cryostat design suitable for low temperature scanning probe microscopy INSTITUTE OF PHYSICS PUBLISHING MEASUREMENT SCIENCE AND TECHNOLOGY pp.859-864

[16] Q. Dai_ R. Vollmery Robert W. Carpickz D. F. Ogletree Miguel Salmerony 1995, A variable temperature ultrahigh vacuum atomic force microscope, Departmental Papers (MEAM) University of Pennsylvania Year 1995 pp.5266-5271

[17] D.H. Agrawal, A.M. Pathan, P.M. Bhatt B.V. Dave, N.V. Bora," A Portable Experimental set-up for AFM to work at cryogenic temperature",NCRTET 2011,Birla Vishvakrama Mahavidhyalay,Vidhyanagar,May-2011 